\& Laves. Of these $4_{1}$ is the diamond net, $4_{3}$ arises by joining up the mid-points of adjacent links in $\mathbf{3}_{1}$ (see Part 1 ), and $4_{4}$ results from placing a tetrahedral group at each point of $4_{1}$. The hexagonal form of $4_{1}$ is the 4-connected Net 6 of Table 2 and Figs. 2 and 11; the hexagonal variant of $4_{2}$ has been illustrated in Fig. 17 as a packing of tetrahedra.

\section{Inert gas hydrate structures}

To complete our discussion of 4-connected nets we mention here the structures which have been proposed (Claussen, $1951 a, b, c)$ for hydrates with formulae close to $X .6 \mathrm{H}_{2} \mathrm{O}$ and $Y .17 \mathrm{H}_{2} \mathrm{O}$ where $X$ is $\mathrm{A}, \mathrm{CH}_{4}, \mathrm{CO}_{2}$, etc. and $Y$ is $\mathrm{CH}_{3} \mathrm{I}, \mathrm{C}_{2} \mathrm{H}_{5} \mathrm{Cl}$, etc. These structures are open packings of water molecules with the inert gas or other material in the interstices. Since each water molecule in an 'expanded ice' framework has four neighbours arranged tetrahedrally the required networks are 4-connected. It might have been expected that the nets would have been found among those in which the links outline polyhedra in the spacefillings of Andreini (Table 3). Instead, these hydrate structures correspond to very elegant space-fillings by (a) pentagonal dodecahedra and 14-hedra (with 12 5-gon and 2 6-gon faces), and (b) pentagonal dodecahedra and 16-hedra (with 125 -gon and 46 -gon faces). The unit cells contain respectively 46 and 136 water molecules.

\section{References}

ANDREINT, A. (1907). Mem. Soc. ital. Sci. nat. (3), 14, 75. Claudssen, W. F. (1951a). J. Chem. Phys. 19, 259.

Cladussen, W. F. (1951b). J. Chem. Phys. 19, 662.

Cradussen, W. F. (1951c). J. Chem. Phys. 19, 1425. Fedorov, E. v. (1904). Z. Kristallogr. 38, 321.

HeEsCH, H. \& LAVES, F. (1933). Z. Kristallogr. 85, 443.

SmITH, J. V. (1953). Acta Cryst. 6, 613.

TAYLOR, W. H. (1933). Z. Kristallogr. 85, 425.

Wescs, A. F. (1954). Acta Cryst. 7, 535.

Acta Cryst. (1954). 7, 554

\title{
An X-ray Microbeam Study of Polycrystalline Specimens of Aluminium and Iron Deformed in Tension
}

\author{
By A. KELLY* \\ Crystallographic Laboratory, Cavendish Laboratory, Cambridge, England
}

(Received 11 March 1954)

\begin{abstract}
Polycrystalline specimens of pure aluminium and of iron have been examined with the X-ray microbeam technique at various stages of a tensile test. From the $X$-ray photographs, measurements have been made of the size of the particles formed within the grains during deformation, of the breadths of the X-ray reflexions from these crystallites and of the mean angle between them. The particles are identified with the regions between slip bands in the material and an attempt is made to account for the stress-strain curve of the specimens from this hypothesis and the results of a dislocation theory due to Frank. Reasonable agreement is found between calculation and experiment.
\end{abstract}

\section{Introduction}

$\mathrm{X}$-ray microbeam methods have been used previously to examine a number of polycrystalline metals after deformation by rolling (Hirsch \& Kellar, 1952; Hirsch, $1952 a, b ;$ Gay \& Kelly, 1953a, b). It was decided to carry out an investigation of specimens of aluminium and iron deformed in tension, first, because the specimens are then in a more precisely defined mechanical state, and second, to test whether a different method of deformation resulted in the development of a different texture within the material.

The interpretation of the earlier results has led to

* Now at Department of Mining and Metallurgical En. gineering, University of Illinois, Urbana, U.S.A. the formulation of a theory to account for the ultimate yield stress of polycrystalline metals containing a particle structure of the type found (Gay, Hirsch \& Kelly, 1954). The results presented in this paper are used in an attempt to extend this theory to explain the work-hardening of the materials in terms of the experimentally observed variation of particle size with plastic strain.

Polycrystalline aluminium has been thoroughly investigated because this material is particularly suitable for examination with the X-ray microbeam technique. Fewer experimental results are available for iron specimens but these are of importance because they confirm the existence of particles of size $\sim 1-2 \mu$ found within the rolled material (Gay \& Kelly, 1953a). 


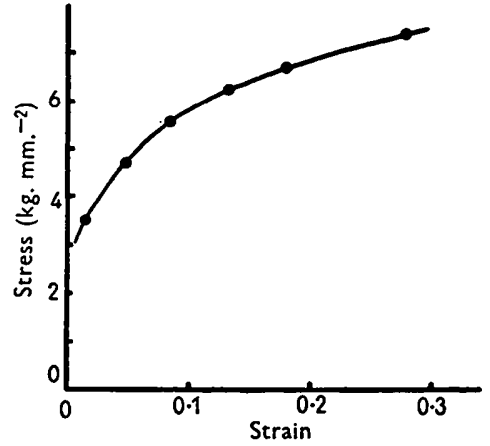

(a)

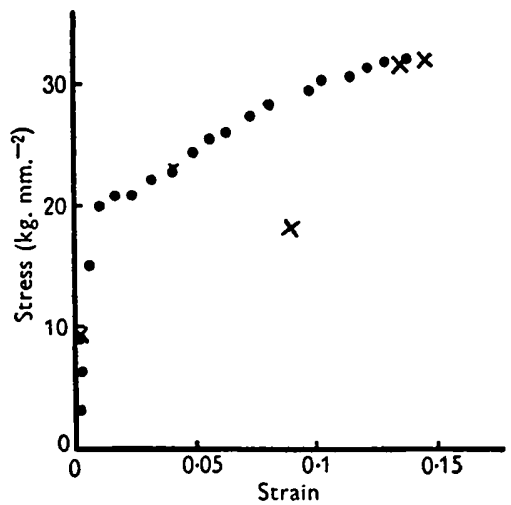

(b)

Fig. 1. (a) Stress-strain curve of an aluminium specimen. (b) Stress-strain curve of an iron specimen. Dots: experimental points; crosses: calculated values (see $\S 6$ ).

\section{Preparation and deformation of specimens}

The aluminium used was of $99.994 \%$ purity, supplied by the Aluminium Wire and Cable Co. Ltd. This material was plunge ground to produce tensile specimens of circular cross-section of diameter $1.5 \mathrm{~mm}$. and of gauge length approximately $1 \mathrm{~cm}$. These test pieces were deformed in a Hounsfield Tensometer. Each specimen was strained the required amount and then unloaded and examined with an $X$-ray beam of appropriate diameter. The strain was measured by the distance between small scratch marks inscribed on the surface of the specimen. The load on the specimen was read directly from the tensometer and corrected to true stress. The specimen was then replaced in the tensometer and the procedure repeated until fracture occurred. The average time between successive strainings was about two days.

The iron specimens were in the form of wires of deoxidized iron supplied by the British Iron and Steel Research Association; these were of $99.96 \%$ purity. The initial gauge length was $10 \mathrm{~cm}$. and the diameter $1.03 \mathrm{~mm}$. The straining and examination of the iron specimens were carried out in the same way as for those of aluminium.

Typical stress-strain curves obtained in this way from specimens of aluminium and iron are shown in Fig. 1.

\section{Examination of specimens}

After straining, the specimens were examined in the centre of the gauge length using $X$-ray back-reflexion methods. The form of the specimens was such that fracture always occurred at the centre of the gauge length. X-ray microbeams of diameter down to $30 \mu$ were used and also a normal $X$-ray beam of diameter $\sim 1 \mathrm{~mm}$. The aluminium specimens were examined with $\mathrm{Cu} K \alpha$ radiation and the 422 ring was examined in detail. Those of iron were examined with $\mathrm{Fe} K \alpha$ radiation, filtered with manganese dioxide, and the 220 ring was examined in detail.

\section{Experimental results for aluminium}

\section{(a) Particle size}

As the material is strained, the original sharp spots obtained on X-ray back-reflexion photographs of the undeformed material spread out into arcs around the Debye-Scherrer ring. By using X-ray microbeams of appropriate diameter, these arcs may be resolved into spots, and an estimate of the particle size may be made by counting the number of spots around the ring (Hirsch \& Kellar, 1952). The spots on the photographs are linked by a continuous background. The X-ray microbeam photographs for aluminium obtained in the present work were very similar to those described by Hirsch \& Kellar for the rolled material.

Fig. 2. shows a plot of the average particle size found within the grains of three specimens of initial grain sizes $27 \mu, 37 \mu$ and $53 \mu$ at various stages of

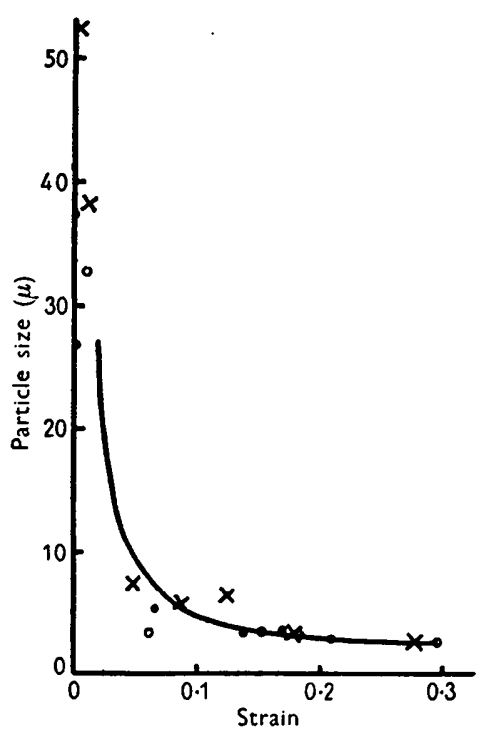

Fig. 2. Experimental variation of particle size with plastic strain for three specimens (dots, crosses and circles) of aluminium. The curve represents the relation $t=a / \varepsilon+c$ $($ see $\S 6)$. 
deformation up to fracture. It is seen that at small strains there is a rapid fall in the average particle size but that after strains of about $15 \%$ the change of particle size with increasing deformation is slow and reaches a lower limit at a value of about $2 \cdot 8 \mu$. The specimens necked down and broke at strains of slightly more than $30 \%$.

\section{(b) Misorientations}

The total angular range of orientation of the material within a deformed grain can be found from the angular extent of the arcs around the Debye-Scherrer ring (Hirsch \& Kellar, 1952). The variation with strain of this total angular misorientation is shown in Table 1.

Table 1. Misorientations in aluminium as a function of strain

$\begin{array}{cc}\text { Strain }(\%) & \begin{array}{c}\text { Total angular misorientation } \\ \text { within one original grain }\left({ }^{\circ}\right)\end{array} \\ 3 & 3 \\ 6 & 8 \\ 13 & 12 \\ 20 & 16 \\ 30 & 18\end{array}$

The values are averages for a number of specimens. It will be seen that the range of misorientation seems to increase up to fracture. It is possible that the apparent continued increase at large strains is not significant since the same grain is not examined after each increment of strain.

\section{(c) Spot shapes}

The lengths of the discrete spots obtained on the microbeam photographs were measured in directions radial and tangential to the Debye-Scherrer ring and the values averaged around the ring. These measurements were interpreted in terms of the quantities $d \varphi_{1}, d \varphi_{2}, d \varphi_{3}$, introduced by Hirsch (1952a), each of which gives an average value of the physical broadening, in a particular direction, of the $\mathbf{X}$-ray reflexion from a particle. The results, averaged for a number of specimens, are given in Table 2 . No value is given for

\section{Table 2. Values of averaged angular breadths as a} function of strain for aluminium

$\begin{array}{cccc}\text { Strain }(\%) & d \varphi_{3}\left(\text { radian } \times 10^{-3}\right) & d \varphi_{1}\left(\text { radian } \times 10^{-3}\right) & \begin{array}{c}Y d \varphi_{1} \cot \theta \\ \left(\mathrm{kg} . \mathrm{mm} .^{-2}\right.\end{array} \\ 6 & 0 \cdot 7 & 1 \cdot 3 & 3 \cdot 6 \\ 13 & 1 \cdot 3 & 1 \cdot 9 & 5 \cdot 3 \\ 20 & 1 \cdot 8 & 2 \cdot 5 & 6 \cdot 7 \\ 30 & 2 \cdot 4 & 2 \cdot 9 & 7 \cdot 8\end{array}$

a strain of less than $6 \%$ since consistent values of spot measurements cannot be obtained from lightly strained specimens.

\section{Experimental results for iron}

As in the case of aluminium, the original sharp spots obtained on back-reflexion photographs spread out into ares around the Debye-Scherrer ring when the material is deformed. By using X-ray microbeams of diameter down to $\sim 60 \mu$ these arcs can be resolved into spots. The spots are much less well resolved from one another than in the case of aluminium and are connected by a strong background. Discrete spots may be observed on photographs from the material extended to fracture, which occurred after about $15 \%$ strain; an example is shown in Fig. 3. A particle size

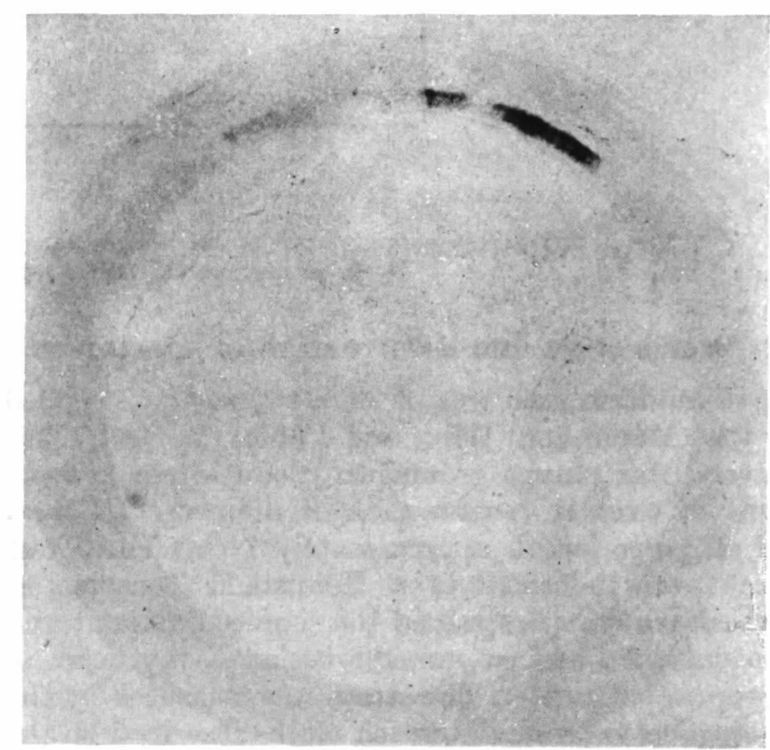

Fig. 3. X-ray microbeam back-reflexion photograph of an iron wire extended to fracture. (Beam diameter $68 \mu ; 220$ ring; $\mathrm{Fe} K \propto$ radiation.)

of $\sim 1.5 \mu$ was found from a spot count on this photograph and this value was checked from photographs of two other specimens and agrees well with that inferred from the trend of the results for rolled iron (Gay \& Kelly, 1953a).

Table 3 shows the averaged results obtained from

Table 3. Measurements of particle sizes, misorientations and averaged angular breadths for iron deformed in tension

$\begin{array}{cccc}\text { Mis- } \\ \text { Strain (\%) } & \text { Particle size }(\mu) & \begin{array}{c}\text { Mienta- } \\ \text { orions }\left({ }^{\circ}\right)\end{array} & d \varphi_{3}\left(\text { radian } \times 10^{-3}\right) \\ \text { tion } & \sim 20 \text { (Grain size) } & - & 1 \cdot 3 \\ 3.9 & 3 & 2 \frac{1}{2} & 2 \cdot 9 \\ 8.9 & 4.9 & 4 & 2 \cdot 7 \\ 13.4 & 1.6 & 6 & 2.5 \\ \text { Broken } & 1.5 & 7 & 3.0\end{array}$

photographs of three specimens. The values obtained for the average total misorientation of material within one deformed grain are similar to those found in rolled iron at comparable deformations. From the measurements of the spot shapes averaged round the ring, values of $d \varphi_{3}$ only were obtained. This is because the divergence of the $\mathrm{X}$-ray beam necessary to obtain good resolution of the photographs is too small to 
Table 4. Values of $\sigma^{2} t$ at various values of the particle size for aluminium deformed in tension

\begin{tabular}{ccc}
\multicolumn{3}{c}{ Specimen annealed $3 \mathrm{hr}$. at $350^{\circ} \mathrm{C}$. } \\
Strain $(\%)$ & Particle size $(\mu)$ & $\sigma^{2} t\left(10^{12}\right.$ dyne $\left.^{2} \mathrm{~cm} \cdot{ }^{-3}\right)$ \\
$27 \cdot 8$ & $2 \cdot 9$ & 157 \\
$18 \cdot 0$ & $3 \cdot 4$ & 150 \\
$13 \cdot 4$ & $6 \cdot 6$ & 239 \\
$8 \cdot 4$ & $6 \cdot 4$ & 192 \\
$4 \cdot 8$ & $7 \cdot 6$ & 166
\end{tabular}

allow unambiguous evaluation of the quantity $d \varphi_{1}$ (Hirsch, 1952a).

\section{Physical interpretation}

Both the mean particle volume and the physical broadening of the X-ray reflexions from the particles have been measured. Application of a treatment due to Hirsch (1952a) shows that part of this broadening must be due to distortion of the particles. If the particles are taken to be elastically strained the stresses necessary to maintain these strains are given by $Y d \varphi_{1} \cot \theta$ ( $Y=$ Young's modulus). These are of the order of the yield strength, as is demonstrated in Table 2 for aluminium if the values of the quantity $Y d \varphi_{1} \cot \theta$ are compared with the curve of Fig. la.

A recent publication (Gay, Hirsch \& Kelly, 1954) discusses the interpretation of the distortion of the particles and the formation of an angle between them. The particles are identified with the regions between slip bands in the material, and the boundary regions between particles are assumed to contain an array of dislocations piled up behind one another against an obstacle in their slip plane.

The condition that the stress concentration due to these dislocations can lead to the static formation of a dislocation loop ahead of the obstacle and hence to yield of the material has been given by Frank (1951). It can be written in the form

$$
\sigma \sim \frac{G}{25} \sqrt{ } \frac{N s}{t}
$$

for material at room temperature. $\sigma$ is equal to the applied stress, $G$ is the rigidity modulus, $s$ is the Burgers vector and $t$ is the length of slip plane in which the dislocations are piled up. $N$ is a number such that the distance $N s$ is equal to the thickness of the barrier plus the size of dislocation loop likely to be formed at room temperature. Gay, Hirsch \& Kelly use this relation to account for the ultimate yield stress of a number of metals by identifying $t$ with the ultimate particle size found from $\mathrm{X}$-ray microbeam examination and taking $N=25$, i.e. a barrier thickness of about $50 \AA$ is assumed.

It is of interest to investigate whether this relation may be used to account for the work hardening of the metals examined in the present experiments, i.e. whether the stress $(\sigma)$ calculated from the above relation may be identified with the yield stress at each stage of the stress-plastic-strain curve if the para-

\begin{tabular}{ccc}
\multicolumn{3}{c}{ Specimen annealed $1 \frac{1}{2} \mathrm{hr}$. at $250^{\circ} \mathrm{C}}$. \\
Strain $(\%)$ & Particle size $(\mu)$ & $\sigma^{2} t\left(10^{12} \mathrm{dyne}^{2} \mathrm{~cm} .^{-3}\right)$ \\
$29 \cdot 8$ & $2 \cdot 9$ & 164 \\
$21 \cdot 0$ & $3 \cdot 1$ & 162 \\
$17 \cdot 0$ & $3 \cdot 8$ & 168 \\
$13 \cdot 8$ & $3 \cdot 5$ & 121 \\
$6 \cdot 4$ & $5 \cdot 5$ & 134
\end{tabular}

meter $t$ is identified with the corresponding particle size. As the materials are strained the value of $t$ is found to vary and it may be that the value of $N$ in equation (1) varies also. If the yield strength $(\sigma)$ when a particle size $t$ has been reached is given by (1) then:

$$
\begin{gathered}
N=625 \sigma^{2} t / G^{2} s, \\
\text { i.e. } \quad N \propto \sigma^{2} t .
\end{gathered}
$$

Table 4 shows the values of $\sigma^{2} t$ obtained in the present experiments for two specimens of aluminium using the measured values of $\sigma$ and of particle size.

The various values of $\sigma^{2} t$ show a large spread, but no consistent change with particle size is apparent. It is seen that the values widely different from the average were obtained from those $\mathrm{X}$-ray photographs giving values of the particle size not in accord with the general trend of the results. It appears, then, that the value of $N$ does not vary greatly with strain, i.e. that the thickness of the barrier which must be penetrated so that further plastic yield can occur is approximately the same at all stages of the deformation. The average value of $\sigma^{2} t$ for both sets of figures in Table 4 is $165 \times 10^{12} \mathrm{dyne}^{2} \mathrm{~cm} \cdot{ }^{-3}$. Using this value in equation (2) we have $N=64$ and we may put

$$
\sigma \sim \frac{G}{3} \sqrt{\frac{s}{t}}
$$

According to this theory, then, the value of the stress necessary for further plastic yield to occur depends only on the size of the particles within the deformed grains, i.e. the average spacing of the barriers.

In Fig. 2 the curve represents the relation:

$$
t=a / \varepsilon+c,
$$

where $\varepsilon$ is the plastic strain, $t$ is the particle size and $a$ and $c$ are constants having the values $0.42 \times 10^{-4} \mathrm{~cm}$. and $1.3 \times 10^{-4} \mathrm{~cm}$., respectively. The experimental points are fairly evenly scattered about this empirical curve. Taking (4) as an approximate representation of the variation of the average particle size with strain, the substitution of (4) in (3), giving

$$
\sigma=\frac{G}{3} \sqrt{a / \varepsilon+c},
$$

should be an approximation to the stress-strain curve. This curve is plotted in Fig. 4 together with the experimental values for the three specimens examined in detail. The curve is not drawn for small values of the 


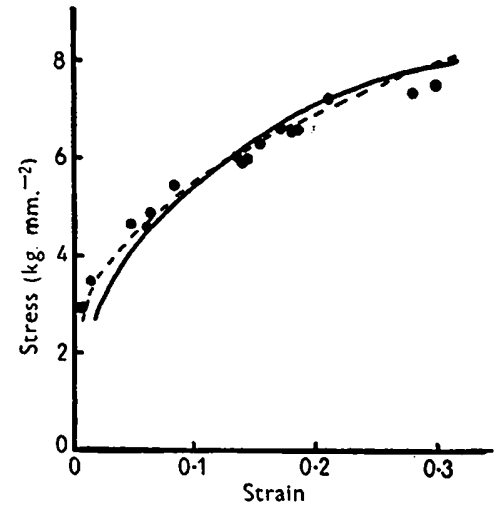

Fig. 4. Experimental variation of stress with strain for three specimens of aluminium (dots represent experimental points). The broken curve represents a parabolic variation of stress with strain; the full curve represents the relation

$$
\sigma=\frac{G}{3} \sqrt{a / \varepsilon+c}
$$

strain since few determinations of particle size were made at a very low strain.

The agreement is good to within an order of magnitude, but a better approximation to the variation of stress with strain is a parabola (see broken curve in Fig. 4). The theoretical curve gives a parabolic relation only at small strains when $a / \varepsilon \gg c$.

Fewer determinations of particle size were made in the case of iron than of aluminium. Values of the yield stress calculated from the measured particle size, at known strains, using equation (3) are compared with the experimental values in Fig. $1(b)$. It is found that equation (3) gives values of the yield stress of the correct order of magnitude. The point far from the experimental curve was obtained from a measurement of particle size not in accord with the trend shown by the others. The very close agreement of the other four points with the experimental values provides strong confirmation that the nature of the variations is correctly given by the theory. This is not affected by the fact that the rigidity modulus was not measured for the material used here, but was taken as that for wrought iron, $7 \cdot 7 \times 10^{11}$ dyne $\mathrm{cm}^{-2}$, for it is the scaling of theory to experiment which is dependent upon the numerical values chosen for this constant and the boundary thickness $N s$ in equation (I).

\section{Discussion}

If the approximate agreement between theory and experiment is significant it means that the parameter ' $t$ ' is representative of the minimum yield stress of the particle. In these experiments the average particle size is obtained as the cube root of the average particle volume measured from the $\mathrm{X}$-ray photographs. This implies that the particles are approximately equiaxed over the whole range of deformation, even at small values of the strain. If the boundaries between particles are formed by slip bands in the material, as Gay, Hirsch \& Kelly (1954) propose, then slip must occur on several systems even at small deformation in these fine-grained polycrystalline specimens.

The shape of the stress-strain curve is accounted for here solely by the variation of particle size with strain. The initial rapid rise of stress with strain is thus due to the rapid fall in particle size, i.e. to the increase in the number of barriers per unit length of slip plane. The flattening of the stress-strain curve at higher strains is due to the approach to a limiting particle size, i.e. to a lower limit to the spacing of the barriers. In this respect the present theory to account for the stress-strain curve of fine grained polycrystalline metals differs from that due to Mott (1953) for the strain hardening in single crystals, since in the latter the stress-strain curve is found to be independent of the spacing of the barriers.

It should be noted that Frank's calculations, as used here to calculate the stress-strain curve from the measured variation of particle size with strain, do not predict a large variation of the stress-strain curve with temperature.

The author is indebted to Prof. Sir Lawrence Bragg and to Dr W. H. Taylor, in whose laboratory this work was carried out, for their constant help and encouragement. He would like to thank Prof. J.S. Koehler (Department of Physics, University of Illinois) for his comments on the manuscript, and his colleague Dr P. B. Hirsch for invaluable assistance on many occasions. Thanks are also due to $\mathrm{Mr}$ G. C. Smith for providing the aluminium specimens and to the British Iron and Steel Research Association for a supply of deoxidized iron. This work was carried out whilst the author was in receipt of a maintenance allowance from the Department of Scientific and Industrial Research.

\section{References}

Frank, F. C. (1951). Pittsburgh Conference on Plastic Deformation of Crystalline Solids p. 89. Office of Naval Research.

GAY, P., Hirsch, P. B. \& Kelly, A. (1954). Acta Cryst. 7, 41 .

GaY, P. \& Kelly, A. (1953a). Acta Cryst. 6, 165.

Gay, P. \& Kelly, A. (1953b). Acta Cryst. 6, 172.

Hirsch, P. B. (1952a). Acta Cryst. 5, 168.

Hrrsch, P. B. (1952b). Acta Cryst. 5, 172.

Hirsch, P. B. \& Kellar, J. N. (1952). Acta Cryst. 5, 162.

Motт, N. F. (1953). Phil. Mag. (7), 44, 742. 